\title{
Adsorption-site mixing at a continuous order-disorder phase transition
}

\author{
C. Schwennicke \\ Institut für Festkörperphysik, Universität Hannover, Appelstraße 2, D-30167 Hannover, Germany \\ M. Sandhoff \\ Institut für Theoretische Physik, Universität Hannover, Appelstraße 2, D-30167 Hannover, Germany \\ W. Sklarek, D. Jürgens, and H. Pfnür \\ Institut für Festkörperphysik, Universität Hannover, Appelstraße 2, D-30167 Hannover, Germany
}

(Received 8 November 1994; revised manuscript received 6 April 1995)

\begin{abstract}
The temperature-dependent probability of occupation of the two threefold-coordinated adsorption sites was determined for the system $\mathrm{S} / \mathrm{Ru}(0001)$ at a coverage of 0.33 , analyzing energy-dependent intensity curves $\left[I(E)\right.$ curves] in low-energy electron diffraction of the ordered $\sqrt{3} \times \sqrt{3} R 30^{\circ}$ phase and of the disordered system. We show that this probability agrees fully with a lattice gas model containing a finite difference in adsorption energy between these sites.
\end{abstract}

\section{INTRODUCTION}

Studies of short-range ordered layers at surfaces have become standard only in recent years as techniques sensitive to short-range order like diffuse low-energy electron diffraction (DLEED) ${ }^{1}$ surface extended x-ray absorption fine structure, ${ }^{2}$ and photoelectron diffraction ${ }^{3}$ become increasingly available and reliable. These studies are aimed towards a more complete understanding of processes on surfaces like growth, diffusion, and chemical reactions on surfaces. In general, structural investigations of systems with only short-range order are more demanding than on those with long-range order, since the adsorption geometry may vary locally (or in time) in a short-range ordered system. Metastability and the occurrence of several different local configurations in case of static disorder may even prevent the determination of a unique local configuration. Therefore, almost all successful and complete determinations of adsorption geometries in two-dimensional systems with frozen-in disorder have been restricted to simple systems with only one adsorption site. ${ }^{4}$ The problem of metastability can be avoided near thermal equilibrium. For disordered systems this requires measurements at elevated temperatures, which generally were considered to be problematic, due to increased thermal background. As we will show below, such measurements can be carried out reliably enough so that the determination of temperature-dependent occupation probabilities of different sites is feasible, at least for simple and well defined systems. Corresponding local timedependent fluctuations are integrated out by the methods mentioned above, so that only the time and space averages are obtained.

Not only is this problem challenging from a methodological point of view, as it requires the determination of small concentrations of adparticles on different sites, it has also important consequences for the viability of lattice gas models ${ }^{5}$ to account for adparticle phase tran- sitions and their critical properties. ${ }^{6-8}$

For our study of temperature-dependent occupation probabilities, we choose a simple and well characterized model system, the $\sqrt{3} \times \sqrt{3} R 30^{\circ}$ phase of atomic sulfur on $\mathrm{Ru}(0001)$, which is completed at a coverage of $0.33 .^{9-11}$ Both the phase diagram ${ }^{9}$ and the geometric structure of several ordered phases of $\mathrm{S} / \mathrm{Ru}(0001)$ have been determined recently. ${ }^{10-14}$ Whereas only the hcp threefold site is occupied in the ordered low coverage $p(2 \times 2)$ and $\sqrt{3} \times \sqrt{3} R 30^{\circ}$ phases, the fcc site also becomes occupied in the domain walls formed above a coverage of $0.33 .^{11,12}$ The spontaneous occupation of the second threefold site above a coverage of 0.33 suggests strongly that also at coverages below 0.33 , the smallest possible excitation causing a change of site is to the fcc site, which seems to be supported by our present investigation. The energetic difference between these sites was estimated recently by Monte Carlo simulation of the phase diagram, using a lattice gas model with mainly repulsive lateral interactions. ${ }^{15,16}$ It is a further aim of this study to show that this model, developed independently by modeling the phase diagram, is indeed in full agreement with the results of the study presented here.

For the structural analysis, we used the energy dependent intensities of diffracted spots in low-energy electron diffraction (LEED), ${ }^{17}$ carrying out a full analysis ${ }^{17,18}$ of the adsorbed layer at each temperature, both for the long-range ordered system as well as for the short-range ordered phase above the order-disorder phase transition using the diffuse intensities. ${ }^{19}$ As will be shown below, the diffuse intensities near the $\bar{K}$ points of the $\sqrt{3} \times \sqrt{3} R 30^{\circ}$ superstructure in the disordered state have the same energy dependence as for the ordered structure at lower temperatures, which proves that inelastically scattered electrons do not have a strong influence on the structural analysis even at temperatures as high as $600 \mathrm{~K}$, in agreement with recent quantitative studies on $\mathrm{Al}(111)$ (Ref. 20) of high resolution inelastic electron scattering. This makes our study feasible. 


\section{EXPERIMENTAL AND DATA ANALYSIS}

All experiments were carried out at a base pressure of $5 \times 10^{-11}$ mbars in a $\mu$ metal shielded UHV chamber. The sample ( $8 \mathrm{~mm}$ diameter, $2.0 \mathrm{~mm}$ thick) was cut parallel to the (0001) plane from a single crystal rod by spark erosion after orientation in a Laue camera and was polished with diamond paste down to a grain size of 0.25 $\mu \mathrm{m}$. The sample was resistively heated with chopped ac current by its mount to the sample holder, which again was mounted on a cryostat. Temperature was measured by two Tungsten-Rhenium thermocouples spot welded to the rim of the sample and stabilized to within $\pm 0.1 \mathrm{~K}$ by a computerized temperature control. After standard cleaning procedures, ${ }^{9}$ cleanliness was tested by Auger measurements.

Sulfur was adsorbed by backfilling the chamber with $5 \times 10^{-9}$ mbar $\mathrm{H}_{2} \mathrm{~S}$ at a sample temperature of $300 \mathrm{~K}$, adjusting the coverage to $0.33 \pm 2 \%$ by comparing the order-disorder phase transition temperatures with Ref. 9 after annealing the layers at $600 \mathrm{~K}$. LEED measurements were done at a series of constant temperatures between 300 and $600 \mathrm{~K}$ concentrating on the temperature range between 400 and $500 \mathrm{~K}$, which contains the order-disorder transition $\left(T_{c} \simeq 430 \mathrm{~K}\right)$. By comparing equivalent integral-order spots, deviations from normal incidence were kept below $0.2^{\circ}$. Normal incidence was tested for each measuring temperature separately. The whole LEED pattern was recorded between 30 and 400 $\mathrm{eV}$ in steps of $1 \mathrm{eV}$ (above $E=100 \mathrm{eV}$ in steps of 2 $\mathrm{eV}$ ), using a cooled slow scan CCD camera, and stored on a hard disk for each energy value. A separate computer program was used to extract the $I(E)$ curves of all visible spots from the recorded images. ${ }^{21}$ Hereby the spot intensity was integrated over circles of constant area in $k$ space around each spot. The background for each spot was determined from a ring surrounding the integration area and subtracted from the intensity inside. Finally, the intensity was weighted with the beam current at this energy. It turned out that variation of the size of the integration area did not change positions and relative intensities of maxima and minima of the $I(E)$ curves, so that this procedure also works for the diffuse intensites of the disordered phase around the $\bar{K}$ points of the $\sqrt{3} \times \sqrt{3} R 30^{\circ}$ structure. Best ratios of signal to background were obtained using an integration area with a radius of $15 \%$ of the distance between (00) and (10) spot.

The theoretical $I V$ curves were calculated using a program package developed by Moritz and co-workers. ${ }^{22,23}$ In order to simulate a statistical occupation of different adsorption sites the average $t$-matrix approximation (ATA) was used in the sulfur overlayer. The outermost two substrate layers were treated as composite layers with three sublayers, respectively, to allow for reconstructions. All other layers were treated as single bravais layers. Layer stacking in this package is done with the layer doubling scheme. For all of our calculations, the full point symmetry of the $\sqrt{3} \times \sqrt{3} R 30^{\circ}$ phase was utilized to save computational time. For the ruthenium atoms up to ten phase shifts for each energy point were used. The Ru phase shifts were calculated using the tabulated ruthenium potential of Moruzzi et al. ${ }^{24}$ Sulfur phase shifts were obtained from a potential calculated for a sulfur solid. Vibrational amplitudes were calculated from layer dependent Debye temperatures, using the Debye integral without further approximation.

The imaginary part of the optical potential was set to $V_{0 i}=3.8\left[E /\left(90 \mathrm{eV}+V_{0}\right)\right]^{1 / 3}$. The real part of the optical potential $V_{0}$ (muffin tin constant) was set to $-7.5 \mathrm{eV}$. For $R$-factor analysis, theoretical $I V$ curves were shifted in $1 \mathrm{eV}$ steps with respect to the experimental curves to obtain the best level of agreement. For our study, we concentrated on the $R$ factors defined by Pendry ${ }^{25}$ and by Zanazzi and Jona. ${ }^{26}$

\section{RESULTS AND DISCUSSION}

A comparison of the experimental data at $300 \mathrm{~K}$ and at $600 \mathrm{~K}$ shows only minor differences for the $I(E)$ curves of the superstructure beams, whereas small peak shifts and changes of intensities are seen for integral-order $I(E)$ curves. This demonstrates that the $I(E)$ curves are still dominated by $\sqrt{3}$-correlated atoms on hcp sites. Nevertheless, the $R$ factors increase as a function of temperature, if the fixed geometry and layer independent Debye temperatures determined for the ordered layer at $200 \mathrm{~K}$ (Ref. 11) are used for comparison with the experimental data. Agreement of the fits to the data at elevated temperatures was considerably improved by adjustment of the Debye temperatures for the topmost layers, and tested for consistency by reiterating the geometric analysis described below and the optimization of the Debye Waller factors. Thus the Pendry $R$ factors were reduced to values still significantly above but comparable to those at room temperature. Only after a first optimization of layer dependent Debye temperatures were we able to discriminate reliably between different occupation probabilities of fcc sites. The Debye temperatures of the sulfur overlayer and the first two Ru layers were optimized using data sets between 400 and $600 \mathrm{~K}$, the highest temperature in our experiments, where maximum sensitivity can be expected. This analysis showed that Debye temperatures, i.e., the effective vibrational amplitudes, had to be chosen differently for the bulk, the first substrate layer and the $\mathrm{S}$ layer. Best agreement (with an uncertainty of about $\pm 10 \%$ ) was achieved with $\Theta_{D_{\mathrm{Ru}}} \approx 310 \mathrm{~K}$ for the first $\mathrm{Ru}$ layer, $\Theta_{D_{\mathrm{Ru} 2}} \approx 420 \mathrm{~K}$ for all deeper layers, and $\Theta_{D_{\mathrm{S}}} \approx 550 \mathrm{~K}$. Interestingly, the optimal Debye temperatures for the $\mathrm{S}$ layer and the first $\mathrm{Ru}$ layer correspond to equal vibrational amplitudes, which are about a factor of 1.34 larger than the Ru bulk amplitudes. This result is in full agreement with the expectation that the vibrational frequencies of $S$ are far above the phonon spectrum of the Ru substrate, so that the thermally excited effective vibrations of the adsorbed $S$ atoms are essentially in phase with the $\mathrm{Ru}$ vibrations. The same layer dependent Debye temperatures were also found in a preliminary data analysis of the $p(2 \times 2)$ structure of $S$ on the same surface, which corroborates this picture. ${ }^{27}$

Initial analysis at $600 \mathrm{~K}$ using the model with only hcp 
site occupation ${ }^{11}$ yielded layer distances $d_{A 1}=1.62 \AA$, $d_{12}=2.16 \AA$, and $d_{23}=2.12 \AA$ between $\mathrm{S}$ and first Ru layer, first and second, and between second and third layers, respectively $\left(d_{\text {bulk }}=2.14 \AA\right)$, which are slightly different from those in Ref. 11. The Pendry $R$ factor, however, is only 0.22 compared to 0.169 for the $300 \mathrm{~K}$ data. We note that the increase of $R_{p}$ as a function of temperature is considerably larger for the integral order than for the fractional-order spots.

These small but significant differences between $I(E)$ curves at 300 and at $600 \mathrm{~K}$ must be caused by the transition to a system with only short-range order at high temperature. Therefore, we further optimized our fit using the following model (see Fig. 1). We assumed that short-range order with $\sqrt{3}$ correlations between hcp sites still prevails even at the highest temperatures studied. This assumption is well justified both by our data and from the correlation lengths measured at the ideal coverage of 0.33 (Ref. 28) up to $600 \mathrm{~K}$. Even at this temperature, correlations over $2 \sqrt{3}$ distances still exist. ${ }^{28}$ As short-range $\sqrt{3}$ correlations for the atoms on the hcp sites seem to dominate at all temperatures considered, the atoms on the fcc sites were assumed to be uncorrelated. As shown in Fig. 1, the three fcc sites next to an hcp site are symmetrically equivalent, and, therefore, must be occupied with the same probability, according to our model. Consequently, the $1 \times 1$ symmetry of the substrate results for the fcc sites. In contrast, locally only $1 / 3$ of the hcp sites are occupied in order to give the (local) $\sqrt{3} \times \sqrt{3} R 30^{\circ}$ superstructure. An additional random occupation of the other hcp sites requires the occupation of next nearest neighbor hcp sites, which is energetically very unfavorable ${ }^{16,29}$ and, therefore, was not included in this analysis. Using this model for the LEED analysis, sulfur layers even at high temperatures can be simulated to a good approximation as ordered $\sqrt{3} \times \sqrt{3} R 30^{\circ}$ layers containing uncorrelated vacancies and $S$ atoms on fcc

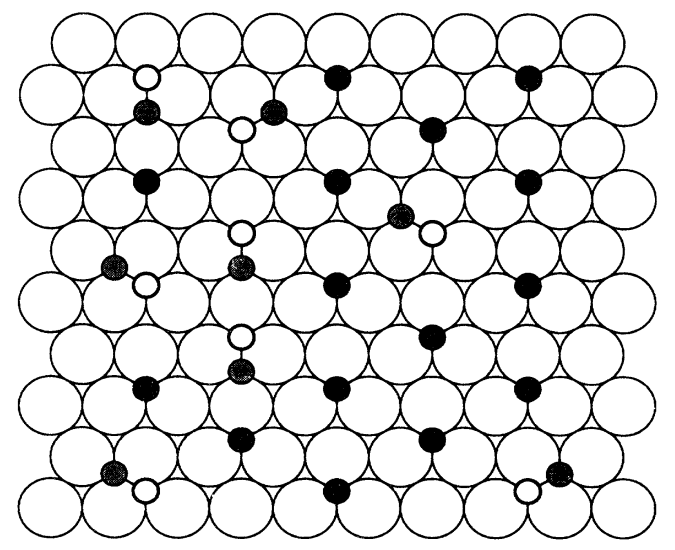

FIG. 1. Hard sphere model of the $\sqrt{3} \times \sqrt{3} R 30^{\circ}$ surface in the short-range ordered phase above $T_{c}$. fcc sites (gray balls) are randomly occupied on the surface. Locally, only one of the three sublattices of hcp sites (black balls) is occupied, due to persistent short-range $\sqrt{3} \times \sqrt{3} R 30^{\circ}$ correlations for the hcp sites. Small open circles are empty sites of the occupied hcp sublattice. sites, because LEED- $I(E)$ analysis is only sensitive to short-range order in any case. ${ }^{17}$ Occupation probabilities of both sites were treated using the ATA for the sulfur layer. ${ }^{30}$

Variation of the occupation probability for fcc sites $p$, $p=\Theta_{\mathrm{fcc}} /\left(\Theta_{\mathrm{hcp}}+\Theta_{\mathrm{fcc}}\right)$, between 0 and 0.9 and of $\Delta z$, the difference in height of $S$ adsorbed in the fcc sites compared to those adsorbed on hcp sites, yielded a best fit to the experimental data at $600 \mathrm{~K}$ at $p$ between 0.2 and 0.3 , depending on the $R$ factor used, and $\Delta z=-0.06 \AA$ (i.e., $\mathrm{S}$ on fcc sites sticks out further). The latter value is comparable to that found in the $c(2 \times 4)(-0.04 \AA)$ (Ref. 13) and $(\sqrt{7} \times \sqrt{7}) R 19.1^{\circ}(-0.03 \AA)$ (Ref. 14) phases.

In Fig. 2, the sensitivity of the Pendry $R$ factor as a function of $p$ is shown separately for integral and fractional-order beams. Obviously, up to $p=0.5$ only the integral-order beams are sensitive to a random occupation of fcc sites. Examples of experimental data for integer and fractional order spots and a comparison with calculated curves for various occupation probabilities $p$ are shown in Figs. 3 and 4, respectively. This insensitivity of fractional-order spots can be easily understood from the $1 \times 1$ symmetry of the fcc sites. Therefore, they do not contribute to the intensity of fractional-order spots in a kinematic approximation. Only a decrease of the intensity of superstructure spots $\sim(1-p)^{2}$ is expected within this approximation. In fact, our own test calculations show that multiple scattering within the adsorbate layer is completely negligible for a coverage of $1 / 3$, thus explaining the mostly unchanged $I(E)$ curves of the superstructure spots. ${ }^{31}$ Thus, our findings are fully consistent with our model of uncorrelated occupation of another site. On the other hand, the essentially unchanged experimental $I V$ curves for fractional order beams as a function of temperature demonstrate that inelastic scattering close to the $\bar{K}$ points in reciprocal space either contain no additional structure, which would modify the $I V$ curves, or its contribution is small enough to make such effects undetectable.

Due to the insensitivity of fractional-order spots to the mixing of additional adsorption sites, the average Pendry

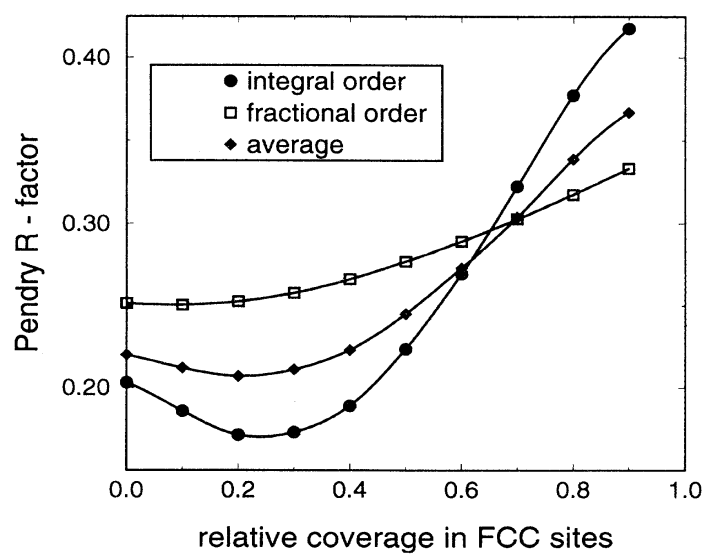

FIG. 2. Pendry $R$ factor as a function of the relative coverage, $p$, in fcc sites separated into that of integral-order spots, fractional-order spots, and an average $R$ factor. 


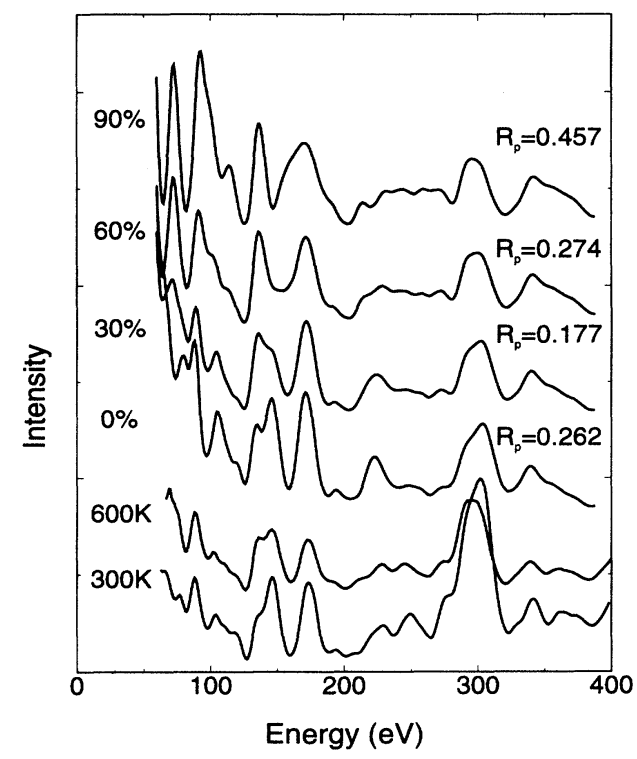

FIG. 3. Experimental $I(E)$ curves for the (10) spot at 300 and $600 \mathrm{~K}$ and calculated $I(E)$ curves for different relative coverages in fcc sites at $600 \mathrm{~K}$. Pendry $R$ factors are calculated with respect to the experimental curve at $600 \mathrm{~K}$.

$R$ factor is only slightly reduced, but $R_{p}$ improves significantly for integral-order beams, as shown in Fig. 3 for the (10) spot. Peak shifts and changes in relative intensities of peaks in the $I(E)$ curve of this beam, evident in experimental data between 300 and $600 \mathrm{~K}$, can be seen to vary systematically as a function of the occupation probability $p$. For the (10) spot alone, the best fit is obtained for $p=0.3$.

For these reasons we used only $I(E)$ curves of the

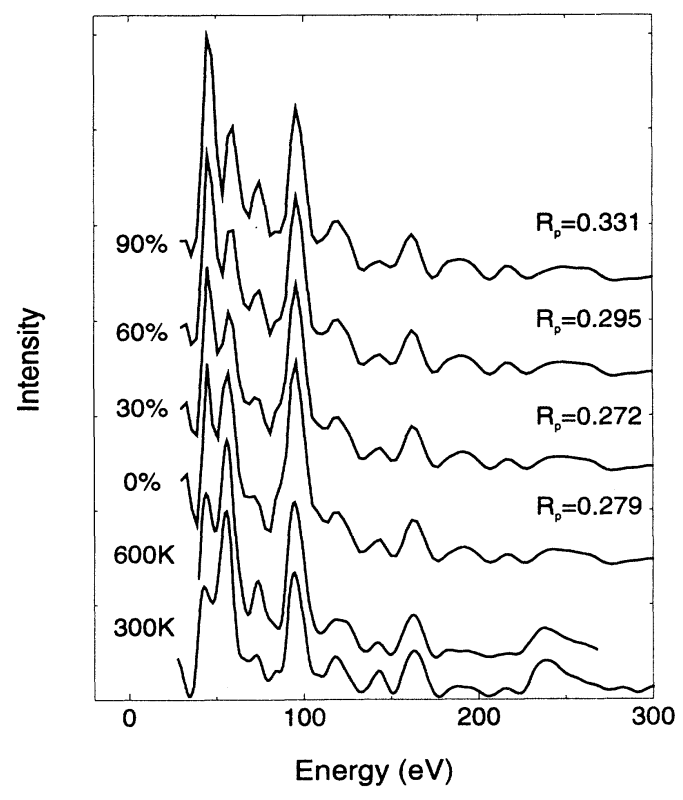

FIG. 4. Same as Fig. 3, but for the $\left(\frac{1}{3}, \frac{1}{3}\right)$ spot. integral-order spots to determine the temperature dependence of the relative coverage of sulfur in fcc sites. Although the improvement of the fits to the high temperature data by our model are still at the limit of significance, they turn out to be completely systematic as a function of temperature, as demonstrated in Fig. 5 using the Pendry $R$ factor. Whereas the minimum remains at $p=0$ for temperatures below $400 \mathrm{~K}$, it shifts to values between 0.2 and 0.3 at temperatures above $450 \mathrm{~K}$ with an increasing tendency for higher temperatures. The same qualitative behavior was obtained by use of the ZanazziJona $R$ factor, which, however, yielded minima at slightly larger occupation numbers for the fcc sites.

The temperature dependence of the absolute coverages in both sites is plotted in Fig. 6. An $S$-shaped behavior of these coverages is found with the maximum slope near the critical temperature of the phase transition. The occupation probabilities of fcc and hcp sites depend both on lateral interactions between $\mathrm{S}$ atoms occupying these sites at various distances, but also on the energetic difference between the hcp and fcc site in a rather complex way, which can only be clarified by simulations. From our experimental results just presented, the use of a lattice gas model containing the two threefold sites seems to be fully justified. We made no attempt, however, to directly fit the experimental data of Fig. 6 as the resulting model might be too ambiguous. Instead we used a lattice gas model already optimized to fit the phase diagram of this system ${ }^{9}$ up to a coverage of $0.5,{ }^{15,16}$ and just plotted the result of this model without further adjustments together with the data in Fig. 6. Qualitatively, and even semiquantitatively, the behavior of the experimentally determined occupation probabilities of hcp and

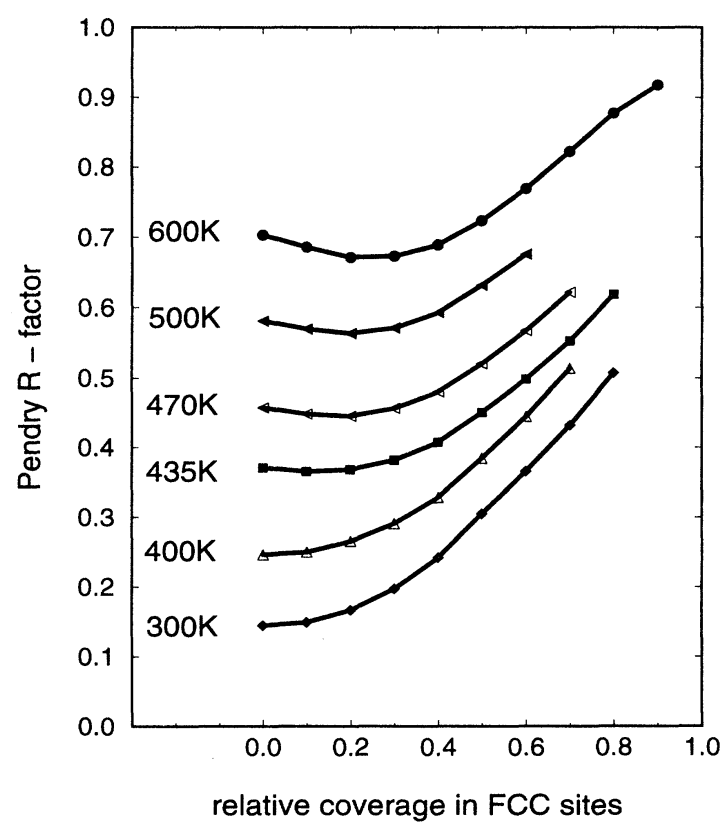

FIG. 5. Pendry $R$ factor for integral-order spots as a function of the relative coverage $p$ in fcc sites for different temperatures. For clarity curves are shifted by $\Delta R_{p}=0.1$. 


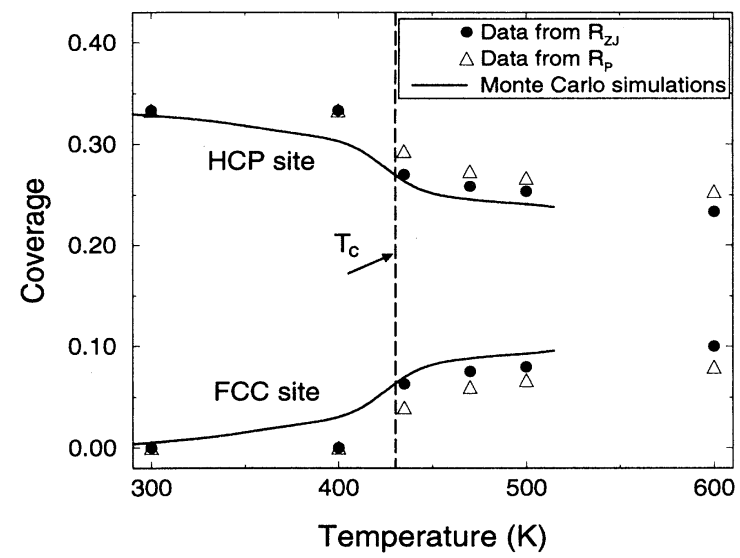

FIG. 6. Temperature dependence of the (absolute) coverage in hcp and fcc sites, using the Pendry $R$ factor and the Zanazzi-Jona $R$ factor of integral-order spots. Lines are results from a Monte Carlo simulation using a lattice gas model (for details, see text).

fcc sites is well reproduced. This model contains five pair interactions and one three particle interaction, and a difference in binding energy between fcc and hcp sites of $0.066 \mathrm{eV}$. The phase diagram contained enough information to adjust these parameters reliably as the low coverage $p(2 \times 2)$ and $\sqrt{3} \times \sqrt{3} R 30^{\circ}$ phases, phase coexistence between $p(2 \times 2)$ and $\sqrt{3} \times \sqrt{3} R 30^{\circ}$, a phase with striped domain walls and the $c(2 \times 4)$ phase, and their respective transition temperatures were available. It is the stability of the striped domain walls, in which the fcc site is occupied also in the ground state, which limits the range for the possible binding energy difference of the two threefold-coordinated sites, so that an occupation of the fcc site was always found even for a coverage of $1 / 3$ in the disordered range.

As expected for a probe of short-range order, ${ }^{32}$ the point of inflection of the distribution function of fcc and hcp sites is at the critical temperature close to $430 \mathrm{~K}$, both in our experiments and in the Monte Carlo simula- tions. The fact that this simulation optimized at totally different physical quantities yields almost quantitative agreement with the experimental data provides further evidence that a lattice gas model gives an appropriate description of the system investigated here.

Our results also show that identical superstructure $I(E)$ spectra above and below the critical temperature $T_{c}$ do not imply identical adsorption geometries in the ordered and disordered phase. We would like to stress that the integral-order spots, which are generally not used in diffuse DLEED experiments, contain considerable relevant information about atoms on the surface adsorbed randomly on lattice sites.

It is interesting to note that the order-disorder transition remains continuous with unchanged threestate Potts exponents both in experiment ${ }^{28}$ and in simulations, ${ }^{16}$ although the second threefold-coordinated site is occupied. This was indeed postulated for the honeycomb lattice if the binding energy difference between the hcp and fcc site was sufficiently strong. ${ }^{33}$

In conclusion, we have presented a structural analysis of a two-dimensional thermally disordered system. Integral order $I(E)$ curves turned out to be sensitive to randomly distributed adsorbed atoms down to a coverage of $4 \%$ of a monolayer (see Fig. 6), whereas superstructure $I(E)$ curves show little change as a function of temperature. The good agreement between our experiment and Monte Carlo simulations, which describe both the phase diagram and the critical properties of the adsorption system, demonstrates that the lattice gas model on a honeycomb lattice is an appropiate and useful description of the thermodynamic properties of the chemisorbed layer of $\mathrm{S} / \mathrm{Ru}(0001)$ at not-too-high coverages.

\section{ACKNOWLEDGMENTS}

This work has been supported by the Deutsche Forschungsgemeinschaft. Helpful discussions with T.L. Einstein and N.C. Bartelt are gratefully acknowledged.
${ }^{1}$ D.K. Saldin, J.B. Pendry, M.A. Van Hove, and D.A. Somorjai, Phys. Rev. B 31, 1216 (1985).

2 J. Stöhr, in X-Ray Absorption: Principles, Applications, Techniques of EXAFS, SEXAFS and XANES, edited by D.C. Koningsberger and R. Prins (Wiley, New York, 1988), p. 443.

${ }^{3}$ D.P. Woodruff, Surf. Sci. 299/300, 183 (1994).

${ }^{4}$ See, e.g., U. Starke, P.L. de Andres, D.K. Saldin, K. Heinz, and J.B. Pendry, Phys. Rev. B 38, 12277 (1988); P. Hu, C.J. Barnes, and D.A. King, ibid. 45, 13595 (1992); H. Wedler, M.A. Mendez, P. Bayer, U. Löffler, K. Heinz, V. Fritsche, and J.B. Pendry, Surf. Sci. 293, 47 (1993).

${ }^{5} \mathrm{~K}$. Binder and D.P. Landau, in Molecule Surface Interaction, edited by K. Lawley (Wiley, New York, 1989), p. 91.

${ }^{6}$ I. Lyuksyutov, A.G. Naumovets, and V. Pokrovsky, TwoDimensional Crystals (Academic Press, New York, 1992).
${ }^{7}$ T.L. Einstein, in Chemistry and Physics of Solid Surfaces VII, edited by R. Vanselow and R.F. Howe (Springer, Berlin, 1987), p. 311.

${ }^{8}$ B.N.J. Persson, Surf. Sci. Rep. 15, 1 (1992).

${ }^{9}$ R. Dennert, M. Sokolowski, and H. Pfnür, Surf. Sci. 271, 1 (1992).

${ }^{10}$ D. Heuer, T. Müller, H. Pfnür, and U. Köhler, Surf. Sci. 297, L61 (1993).

${ }^{11}$ D. Jürgens, G. Held, and H. Pfnür, Surf. Sci. 303, 77 (1994).

12 T. Müller, D. Heuer, H. Pfnür, and U. Köhler, Surf. Sci. (to be published).

${ }^{13}$ C. Schwennicke, D. Jürgens, G. Held, and H. Pfnür, Surf. Sci. 316, 81 (1994)

${ }^{14}$ W. Sklarek, C. Schwennicke, D. Jürgens, and H. Pfnür, Surf. Sci. (to be published). 
${ }^{15}$ M. Sandhoff, Dissertation, Universität Hannover, 1994.

${ }^{16}$ M. Sandhoff, H. Pfnür, and H.-U. Everts (unpublished).

${ }^{17}$ M.A. Van Hove, W.H. Weinberg, and C.M. Chan, LowEnergy Electron Diffraction, Springer Series in Surface Sciences Vol. 6 (Springer, Berlin, 1986).

${ }^{18}$ J.B. Pendry, Low Energy Electron Diffraction (Academic Press, London, 1974).

${ }^{19}$ K. Heinz, U. Starke, and F. Bothe, Surf. Sci. 243, L70 (1991).

${ }^{20}$ A. Büssenschütt and M. Henzler (unpublished).

${ }^{21}$ G. Held, H. Pfnür, and D. Menzel, Surf. Sci. 271, 21 (1992).

${ }^{22}$ H. Over, U. Ketterl, W. Moritz, and G. Ertl, Phys. Rev. B 46, 15438 (1992).

${ }^{23}$ G. Kleinle, W. Moritz, and G. Ertl, Surf. Sci. 238, 119 (1990).
${ }^{24}$ V.L. Moruzzi, J.F. Janak, and A.R. Williams, Calculated Electronic Properties of Metals (Pergamon, New York, 1978).

${ }^{25}$ J.B. Pendry, J. Phys. C 13, 937 (1980).

${ }^{26}$ E. Zanazzi and F. Jona, Surf. Sci. 62, 61 (1977).

${ }^{27} \mathrm{C}$. Schwennicke and $\mathrm{H}$. Pfnür (unpublished).

${ }^{28}$ M. Sokolowski and H. Pfnür, Phys. Rev. B 49, 7716 (1994).

${ }^{29}$ M. Sandhoff, H. Pfnür, and H.-U. Everts, Europhys. Lett. 25, 105 (1994).

${ }^{30}$ F. Jona, K.O. Legg, H.D. Shih, D.W. Jepsen, and P.M. Marcus, Phys. Rev. Lett. 40, 1466 (1978).

${ }^{31} \mathrm{C}$. Schwennicke and H. Pfnür (unpublished).

${ }^{32}$ N.C. Bartelt, T.L. Einstein, and L.D. Roelofs, Surf. Sci. 149, L47 (1984).

${ }^{33}$ E. Domany and M. Schick, Phys. Rev. B 20, 3828 (1979). 